\title{
Postoperative Lemierre's syndrome: a previously unreported complication of transoral surgery. Illustrative case
}

\author{
Giuseppe Mariniello, MD, PhD, Sergio Corvino, MD, Giuseppe Teodonno, MD, Serena Pagano, MD, and Francesco Maiuri, MD \\ Department of Neurosciences, Reproductive and Odontostomatological Sciences, Neurosurgical Clinic, School of Medicine, University "Federico II," Naples, Italy
}

BACKGROUND Lemierre's syndrome is a rare but potentially life-threatening clinical condition characterized by bacteremia and thrombophlebitis of the internal jugular vein, usually secondary to oropharyngeal infection and often caused by Fusobacterium necrophorum; rarely, it occurs after surgical procedures. The most common clinical presentation includes acute pharyngitis, high fever, and neck pain. The diagnosis is based on blood culture and cranial and cervical spine computed tomography (CT)/magnetic resonance imaging (MRI) with contrast. Antibiotic therapy for 3-6 weeks is the mainstay of treatment, while the use of anticoagulant drugs is controversial.

OBSERVATIONS The authors describe a case of Lemierre's syndrome that occurred after transoral surgery. The patient underwent a combined surgical approach from above (transoral) and below (anterolateral transcervical) to the upper cervical spine for the resection of a large anterior osteophyte causing dysphagia, globus sensation, and dysphonia. Three weeks after the surgical procedure, she developed fever and severe neck pain.

LESSONS The aim of this paper is to consider Lemierre's syndrome as a possible complication after the transoral approach, underlining the importance of its early diagnosis and with a suggestion to perform cranial and cervical spine CT or MRI venous angiography in patients who undergo surgery with a transoral approach and exhibit local or systemic signs of infection such as neck pain, persistent fever, and positive blood culture results.

https://thejns.org/doi/abs/10.3171/CASE20118

KEYWORDS Lemierre's syndrome; transoral approach; cervical osteophyte

Lemierre's syndrome (LS), also known as postanginal septicemia or necrobacillosis, first described by André Lemierre in 1936, ${ }^{1}$ is a rare but potentially life-threatening clinical condition characterized by bacteremia and thrombophlebitis of the internal jugular vein (IJV), usually secondary to oropharyngeal infection. ${ }^{2,3}$ It is caused mainly by Fusobacterium necrophorum, ${ }^{2}$ less frequently by streptococci, and rarely by Streptococcus constellatus. ${ }^{4-7}$ Postoperative cases of LS have also been described consequent to surgical procedures for ear, nose, and throat (ENT) tumors or oral cavity pathologies. ${ }^{7}$ The classic triad described by Vogel and Horger $^{3}$ is not always present, and clinical manifestation may vary. Typical cases are characterized by fever, sore throat, and neck pain, while sometimes distant complications may be the only clinical presentation.
The diagnostic criteria and treatment protocol for LS are not fully clarified ${ }^{2,7-17}$ because of the rarity of the syndrome and the absence of related pathognomonic symptoms. The diagnosis is based mainly on blood culture and cervical spine computed tomography (CT)/magnetic resonance imaging (MRI) with contrast to detect bacteremia and IJV thrombosis. Antibiotic therapy for 3-6 weeks represents the mainstay of treatment, while the use of anticoagulant drugs is controversial. ${ }^{18-21}$ Surgery is reserved for selected cases. ${ }^{22-24}$

We report what is, to the best of our knowledge, the first case of LS to occur after transoral surgery, with the aims being to recommend consideration of the possibility of this complication after this kind of surgery and to keep in mind that it is fatal if not treated adequately and in a timely manner. $1,15,16,25,26$

ABBREVIATIONS CT = computed tomography; ENT = ear, nose, and throat; IJV = internal jugular vein; LS = Lemierre's syndrome; MRI = magnetic resonance imaging; $P O D=$ postoperative day; $\mathrm{WBC}=$ white blood cell.

INCLUDE WHEN CITING Published April 26, 2021; DOI: 10.3171/CASE20118.

SUBMITTED November 16, 2020. ACCEPTED December 17, 2020.

(C) 2021 The authors, CC BY-NC-ND 4.0 (http://creativecommons.org/licenses/by-nc-nd/4.0/). 


\section{Illustrative Case}

A 72-year-old woman presented with a 1-year history of progressive dysphagia, especially in swallowing solid foods, globus sensation, and dysphonia, with worsening of clinical symptoms in the last 3 months. CT of the cervical spine showed a large anterior osteophyte (15 $\mathrm{mm}$ at its greater anteroposterior diameter) spanning from $\mathrm{C} 1$ to the upper edge of the $\mathrm{C} 4$ body and causing pharyngoesophageal tract compression (Fig. 1A).

During the patient's hospital stay, oral cavity inspection showed an anterior bulging of the posterior oropharyngeal wall pushing forward to touch the posterior surface of the soft palate and the base of the tongue (Fig. 1B). These data were then confirmed by an oropharyngeal endoscopic transnasal examination performed by an ENT surgeon.

An oropharyngeal barium contrast swallow study revealed a filling defect along the pharynx with lateral displacement of the esophagus (Fig. 1C), confirming the diagnosis of pharyngoesophageal and laryngeal compression. In addition, MRI of the cervical spine showed disc herniation at the $\mathrm{C} 2-3$ level (Fig. 1D).

After a tracheostomy was performed, the patient received antibiotic prophylaxis (cefazolin $1 \mathrm{~g}$ twice daily starting 2 hours before surgery). The patient then underwent microsurgical osteophytectomy with the aid of a high-speed drill through a two-stage surgical approach from above and below directly to the upper cervical spine. The transoral approach (before) was direct to the C1-2 segment, and the anterolateral transcervical approach (after) was direct to the portion extending to $\mathrm{C} 3$ and $\mathrm{C} 4$ (Fig. 2).
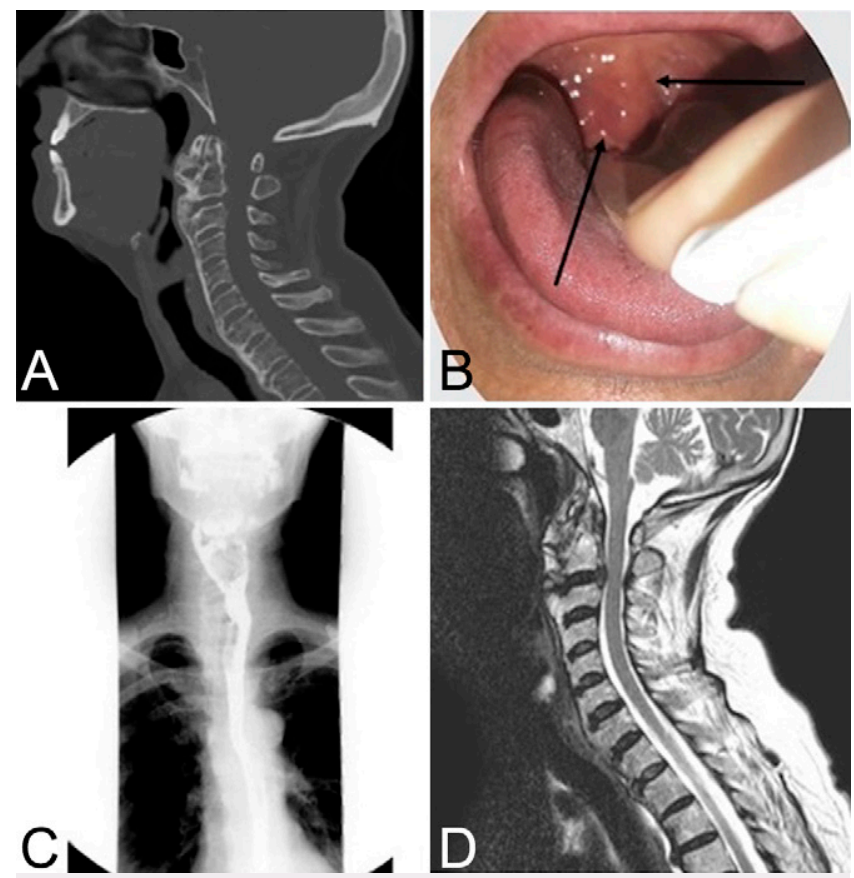

FIG. 1. A: Preoperative CT of the cervical spine showing large osteophyte spanning from $\mathrm{C} 1$ to superior edge of $\mathrm{C} 4$ body. B: Oral cavity examination: macroscopic view of anterior bulging of the posterior oropharyngeal wall (black arrows). C: Preoperative barium contrast swallow showing defect of the filling along the oropharyngeal segment and lateral displacement of the esophagus. D: Preoperative MRI of the cervical spine, sagittal sequence, showing disc herniation at C2-3 level.

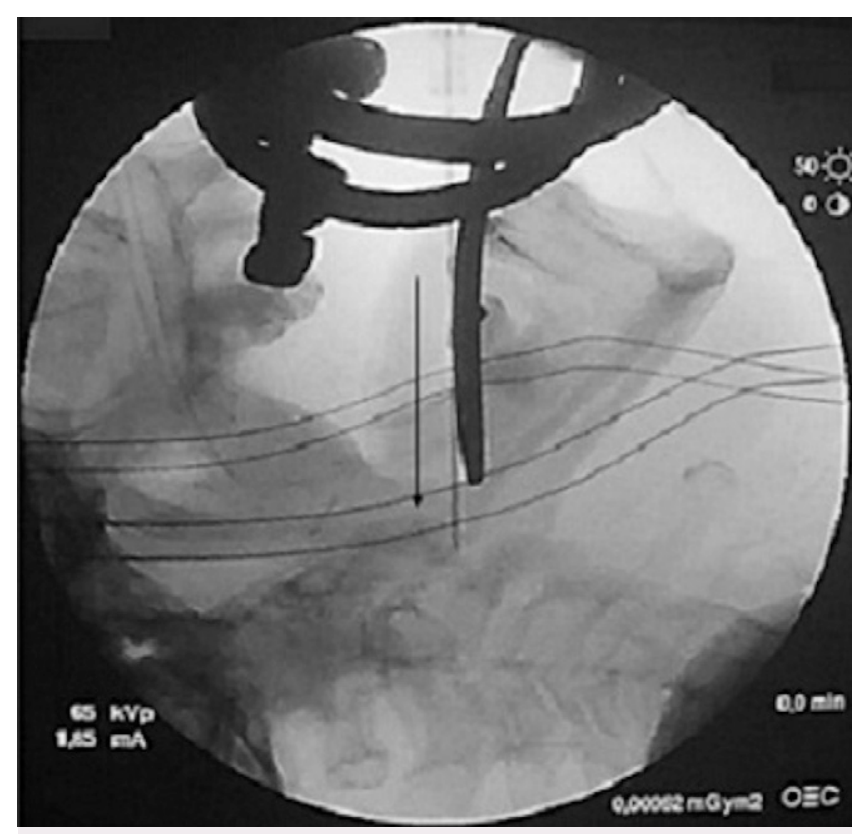

FIG. 2. C-arm radiographic intraoperative fluoroscopy of the cervical spine. The black arrow shows surgical target area of transoral approach (C1-2 vertebral osteophyte).

At the end of the procedure, the patient was transferred to the intensive care unit for a protected awakening. A cervical CT scan (Fig. 3) on postoperative day (POD) 1 showed satisfactory resection of the osteophyte. Thus, parenteral nutrition was administered for the first 4 days, followed by a liquid diet (i.e., juice, soup) for another 2 days, and finally a normal diet was allowed from the 7th POD. Antibiotic therapy per protocol (cefazolin $1 \mathrm{~g}$ twice daily for 3 days) was continued. During the hospital stay, the patient reported a significant improvement of clinical symptoms, and she was discharged on POD 10 apparently without complications.

Three weeks later, because of the onset of severe neck pain aggravated by extension and rotation movements and fever (temperature $39.2^{\circ} \mathrm{C}$ ), the patient was readmitted to our division. Serum studies revealed a white blood cell (WBC) count of $16.8 \times 10^{3} \mu \mathrm{L}$, and a C-reactive protein level of $351 \mathrm{mg} / \mathrm{L}$, and blood culture results were positive for $S$. constellatus. Thus, an appropriate antibiotic therapy (vancomycin $1 \mathrm{~g}$ twice daily and piperacillin-tazobactam $4 \mathrm{~g} / 0.5 \mathrm{~g}$ three times daily), as recommended by an infectious disease specialist and tailored to the culture results, was administered. The patient's echocardiogram excluded the presence of infective endocarditis. Her chest radiograph was normal. Her postcontrast cranial and cervical spine MRI (Fig. 4A) showed good resection of the anterior osteophyte with restoration of the lumen of the pharyngoesophageal tract; diffuse inflammatory hyperintensity of the prevertebral tissues at the C1-3 segment and inhomogeneous contrast enhancement at the posterior cervical muscles (Fig. 4A) were also detected. The angiographic MRI sequences showed that thrombophlebitis of the right IJV extended to the homolateral transverse-sigmoid sinuses (Fig. 4B and C). This finding was confirmed by Doppler ultrasound of the neck veins. Hence, anticoagulant therapy with heparin (4000 IU twice daily) was administered. During the following days, progressive amelioration of the 


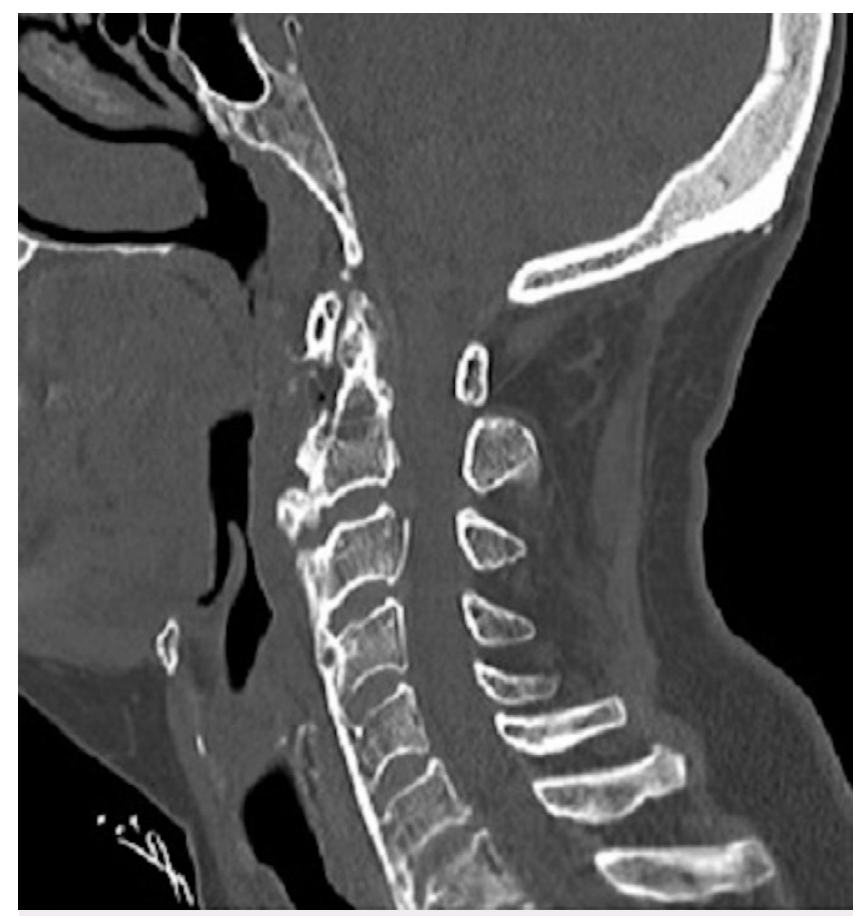

FIG. 3. Postoperative CT of the cervical spine showing satisfactory resection of the large osteophyte and pharyngoesophageal tract's decompression.

patient's general condition was observed, with normalization of inflammation indexes (C-reactive protein, WBC count) and disappearance of the patient's increased temperature and her neck pain.

One week later, a control Doppler ultrasound of the neck veins showed almost complete flow rehabilitation (Fig. 5), and the patient was discharged without neck pain and fever, with a recommendation to continue the pharmacological therapy for another 5 weeks.

\section{Discussion}

LS, referred to as a "forgotten disease" in the postantibiotic era, is a rare (3.6 cases per million people per year ${ }^{27,28}$ ) but potentially life-threatening clinical condition with a mortality rate decrease from $90 \%{ }^{1}$ to $2 \%-5 \%, 18,28$ with the advent of antibiotic therapy.

Microorganisms of the oral cavity flora usually invade the pharyngeal mucosa, already offended by viral or bacterial pharyngitis, extending to the lateral pharyngeal spaces and leading to IJV thrombophlebitis, abscess formation, and systemic emboli. When sustained by $S$. constellatus, ${ }^{4-7}$ as in our case, the disease shows a more aggressive and severe natural course $e^{4,9}$ with tendencies for invasiveness and abscess formation., ${ }^{49}$

Rarely, the infection is consequent to surgical procedures for ENT tumors or oral cavity pathologies, as described in a recent systematic review, ${ }^{7}$ but no cases after neurosurgical procedures have been reported in the literature. The transoral surgical route through the opening of the posterior wall of the oropharynx allows oral cavity microorganisms direct access to the vertebral and paravertebral spaces.

The infection rate related to this surgical approach during antibiotic prophylaxis ranges from $0.6 \%$ to $4 \% .^{30,31}$ The rate of postoperative complications is $21.4 \%$, and the most frequent complications are infection and breakdown of the pharyngeal wound, ${ }^{30,32-34}$ with rates in the literature ranging from $0.1 \%$ to over $18 \%,{ }^{31,33-36}$ mostly within the first 4 months ${ }^{30}$ and mainly affecting patients with rheumatic diseases. ${ }^{30,37}$ Other complications include vertebral artery injury, cerebrospinal fluid fistula, sepsis, malocclusion, injury to the hypoglossal and lingual nerves, mandibular pseudoarthrosis and osteonecrosis, periodontal disease, temporomandibular joint dysfunction, conductive hearing loss and serous otitis media, and swallowing and speech difficulties, ${ }^{38}$ potentially leading to death, ${ }^{39}$ whereas LS as a possible complication after the transoral approach has never been reported in the literature. The risk of complications increases with operative time $>4$ hours and length of stay $>5$ days. ${ }^{40}$ The mortality rate after the transoral approach ranges from $0 \%$ to $8 \%{ }^{34,35,40,41}$ The surgeon who chooses the transoral route has to keep in mind the possibility of infection from oral cavity flora microorganisms. To reduce this risk, we adopted some simple measures: prior to surgery, through an
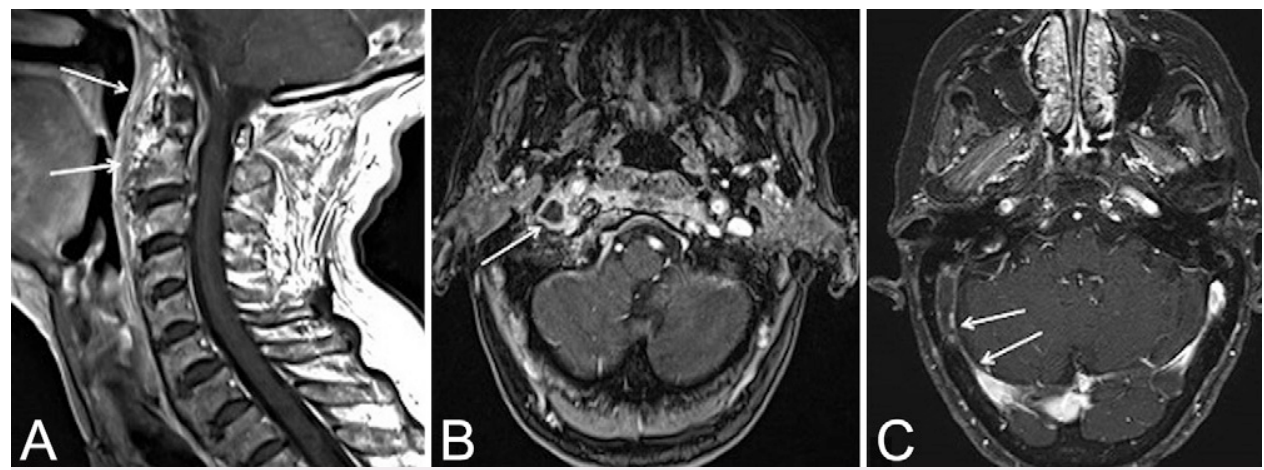

FIG. 4. A: Postoperative MRI with contrast of the skull and cervical spine showing good resection of the anterior osteophyte, with diffuse inflammatory hyperintensity of the prevertebral tissues at C1-3 (white arrows) and posterior cervical muscles with inhomogeneous contrast enhancement. B and C: Postoperative MRI with contrast showing thrombophlebitis of the right IJV extended to the homolateral transverse-sigmoid sinuses (white arrows). 


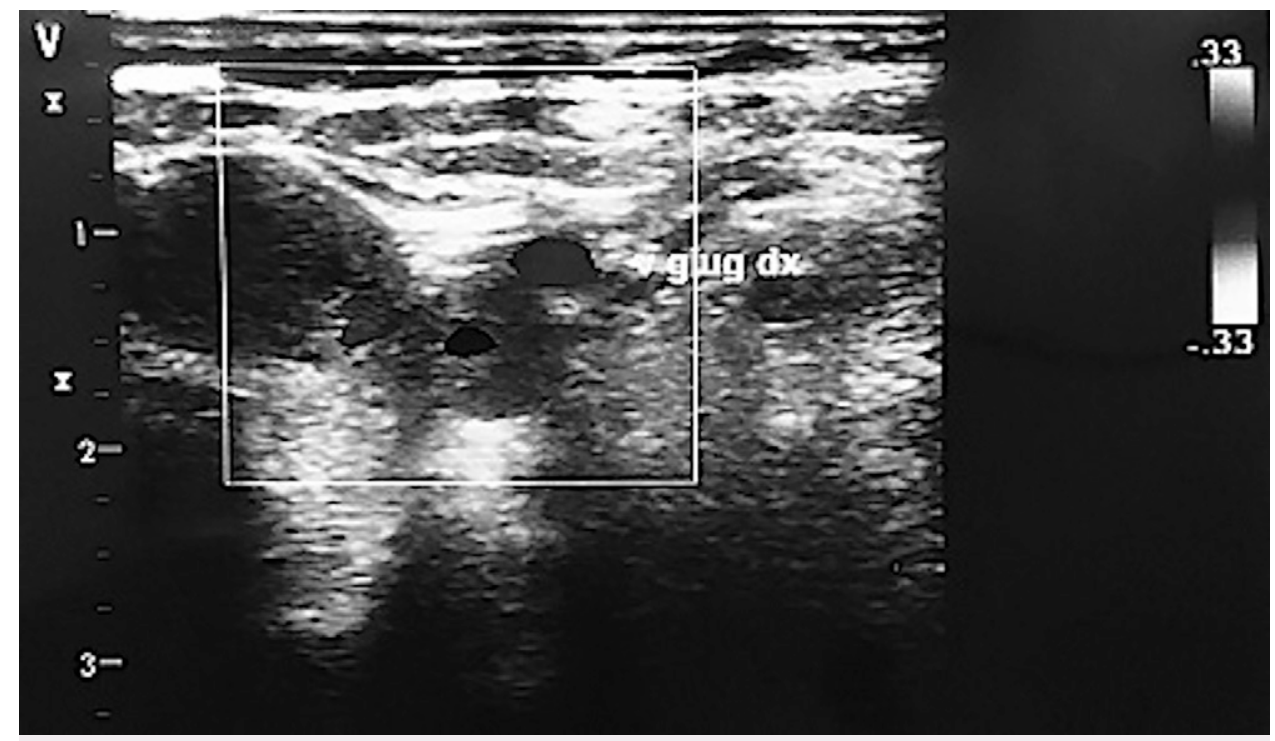

FIG. 5. Doppler ultrasound of the right jugular vein after 1 week of heparin anticoagulant therapy showing an almost complete flow rehabilitation.

accurate investigation of oral cavity hygiene by an oral health specialist, administering mouthwash with $0.05 \%$ chlorhexidine three times per day on the days before surgery; during the surgery, through disinfection of the mouth, pharynx, and nasal cavity with povidone-iodine before mucosal incision; and at the end of the procedure, with the use of a topical antibiotic-impregnated sheath and particular attention to muscular and mucosal plane closure through an interrupted onelayered deep resorbable suture. Also, with regard to performing the tracheostomy, which is not routinely done by all surgeons, we consider, in agreement with Di Lorenzo, ${ }^{33}$ that it helps provide oral antisepsis.

Early diagnosis and appropriate, timely treatment of LS are crucial because the disease's course is rapid and irreversible ${ }^{25}$ and can lead to persistent neurological deficits or death if not adequately treated. $1,15,16,26$

Ultrasound is the first instrumental diagnostic method when IJV is suspected, ${ }^{42}$ followed by CT or MRI with contrast. Considering the onset of clinical symptoms in the postoperative period in our patient, we decided to start performing MRI with gadolinium (Fig. 4) to exclude any surgical complications. The finding of diffuse hyperintensity of the paravertebral tissues at the $\mathrm{C} 1-3$ segment with inhomogeneous contrast enhancement was consistent with the initial phase of a suppurative inflammatory process, with possible evolution to abscess formation if not treated in a timely manner. Thrombophlebitis of the right IJV extending to the homolateral transversesigmoid sinuses (Fig. 4B and C) was treated with low-molecularweight heparin and monitored through ultrasound of the neck veins.

Treatment includes antibiotic and anticoagulant therapy and surgery. ${ }^{11}$ Prompt antibiotic therapy based on blood cultures represents the mainstay of treatment; the optimal duration is variable, from 2 to 20 weeks, depending on the severity of the disease, ${ }^{7,8,12-14}$ but it should last at least until the resolution of clinical symptoms and radiological findings, ${ }^{7}$ with a mean duration of 5 weeks. Antibiotic therapy can be prolonged for 3-6 weeks in the case of a massive fibrin clot and/or abscess ${ }^{17}$ to obtain complete penetration of the drugs.

Timely and adequate antibiotic therapy based on an antibiogram allowed us to obtain normalization of laboratory test results, improvement of clinical symptoms in a few days, and arrest of the progression to abscess formation. 4,15,16,29

The use and the duration of anticoagulant drugs are controversial, ${ }^{18-21}$ even if most studies report a significant improvement of the mortality rate after anticoagulation, ${ }^{43,44}$ especially when intracranial thrombophlebitis is present, to prevent permanent neurological disability, as reported in a recent systematic review, ${ }^{7}$ and septic embolic events arising from IJV thrombosis. ${ }^{15}$ Also, the duration of anticoagulation is a matter of discussion, with recommendations varying from 3-4 weeks to $3-12$ months. ${ }^{45}$ The drug most often used is low-molecular-weight heparin.

Finally, surgical intervention is reserved for cases complicated by an abscess to be drained or in the form of ligation or resection of the IJV, although it is rarely indicated and reserved for cases of persistent septic embolization despite pharmacological treatment. ${ }^{22-24}$

\section{Observations}

This is the first reported case of LS that occurred as a complication of the transoral approach.

\section{Lessons}

The aim of this paper is to recommend consideration of LS as a possible complication after the transoral approach, stressing the importance of its early diagnosis and suggesting exploration by cranial and cervical spine CT or by magnetic resonance venous angiography in patients operated on with this approach who exhibit signs of local or systemic infection. If not treated in a timely and appropriate manner, it leads to persistent neurological deficits and even death. 


\section{References}

1. Lemierre A. On certain septicæmias due to anaerobic organisms. Lancet. 1936;1:701-703.

2. Hong $P$, MacCormick J, Lamothe $A$, Corsten $M$. Lemierre syndrome: presentation of three cases. J Otolaryngol. 2005;34(5):352-358.

3. Vogel M, Horger M. Lemierre syndrome. Article in German. Rofo. 2005;177(1):1-3.

4. Shimada M, Morinaga Y, Kitazaki T, et al. A severe case of Lemierre syndrome with Streptococcus constellatus infection. Jpn J Infect Dis. 2014;67(6):488-489.

5. Benhayoun M, Llor J, Van-Den-Abbeele T, et al. Bilateral jugular thrombosis in Lemierre syndrome. Article in French. Arch Pediatr. 2003;10(12):1071-1074.

6. Righini CA, Karkas A, Tourniaire R, et al. Lemierre syndrome: study of 11 cases and literature review. Head Neck. 2014;36(7):1044-1051.

7. Moretti M, De Geyter D, Goethal L, Allard SD. Lemierre's syndrome in adulthood, a case report and systematic review. Acta Clin Belg. Published online February 26, 2020. doi: 10.1080/ 17843286.2020.173166

8. Johannesen KM, Bodtger U. Lemierre's syndrome: current perspectives on diagnosis and management. Infect Drug Resist. 2016;9:221-227.

9. Olson KR. Case 36-2014: a woman with fever, pharyngitis, and double vision. N Engl J Med. 2015;372(6):581-582.

10. Zhao A, Samannodi M, Tahir M, et al. Lemierre's syndrome: case report and brief literature review. IDCases. 2017;10:15-17.

11. Blessing K, Toepfner N, Kinzer S, et al. Lemierre syndrome associated with 12th cranial nerve palsy-a case report and review. Int J Pediatr Otorhinolaryngol. 2013;77(9):1585-1588.

12. Leoncini E, Ricciardi W, Cadoni G, et al. Adult height and head and neck cancer: a pooled analysis within the INHANCE Consortium. Eur J Epidemiol. 2014;29(1):35-48.

13. Holm K, Svensson PJ, Rasmussen M. Invasive Fusobacterium necrophorum infections and Lemièrre's syndrome: the role of thrombophilia and EBV. Eur J Clin Microbiol Infect Dis. 2015;34(11): 2199-2207.

14. Hansberry DR, D'Angelo M, Prabhu AV, et al. Lemierre's syndrome: acute oropharyngeal infection leading to septic thrombophlebitis of the internal jugular vein with pulmonary septic emboli. Interdiscip Neurosurg. 2020;19:1005773.

15. Walkty A, Embil J. Lemierre's syndrome. N Engl J Med. 2019; 380(12):e16.

16. Teng HW, Chen CY, Chen HC, et al. Fusobacterium septicemia complicated by cerebral subdural and epidural empyemas: a rare case of Lemierre syndrome. J Emerg Med. 2012;43(4):671-673.

17. Dalen CT, Mekhail AM. Lemierre syndrome: early recognition and management. CMAJ. 2015;187(16):1229-1231.

18. Karkos PD, Asrani S, Karkos CD, et al. Lemierre's syndrome: a systematic review. Laryngoscope. 2009;119(8):1552-1559.

19. McGouran D, Keene A, Walklin R, Carter J. A complex case of bilateral Lemierre syndrome with suggestions on anticoagulation management. Intern Med J. 2013;43(6):728-730.

20. Phua CK, Chadachan VM, Acharya R. Lemierre syndrome-should we anticoagulate? A case report and review of the literature. Int $J$ Angiol. 2013;22(2):137-142.

21. Cupit-Link MC, Nageswara Rao A, Warad DM, Rodriguez V. Lemierre syndrome: a retrospective study of the role of anticoagulation and thrombosis outcomes. Acta Haematol. 2017;137 (2):59-65.

22. Chirinos JA, Lichtstein DM, Garcia J, Tamariz LJ. The evolution of Lemierre syndrome: report of 2 cases and review of the literature. Medicine (Baltimore). 2002;81(6):458-465.

23. Bahall M, Giddings S, Bahall K. Lemierre's syndrome: forgotten, but not absent. BMJ Case Rep. 2017;2017:bcr2017221203.
24. Lemierre A, Gregoire R, Laporte A, Couvelaire R. Les aspects chirurgicaux des infections a Bacillus funduliformis. Acad Med. 1938;6:352-359.

25. Lee WS, Jean SS, Chen FL, et al. Lemierre's syndrome: a forgotten and re-emerging infection. J Microbiol Immunol Infect. 2020;53(4):513-517.

26. Kuppalli K, Livorsi D, Talati NJ, Osborn M. Lemierre's syndrome due to Fusobacterium necrophorum. Lancet Infect Dis. 2012;12(10): 808-815.

27. Liu YC, Lee WJ. Lemierre's syndrome: a possible cause of neck pain. Intern Emerg Med. 2019;14(8):1339-1340.

28. Hagelskjaer Kristensen L, Prag J. Lemierre's syndrome and other disseminated Fusobacterium necrophorum infections in Denmark: a prospective epidemiological and clinical survey. Eur J Clin Microbiol Infect Dis. 2008;27(9):779-789.

29. Claridge JE III, Attorri S, Musher DM, et al. Streptococcus intermedius, Streptococcus constellatus, and Streptococcus anginosus ("Streptococcus milleri group") are of different clinical importance and are not equally associated with abscess. Clin Infect Dis. 2001;32(10):1511-1515.

30. Shousha M, Mosafer A, Boehm H. Infection rate after transoral approach for the upper cervical spine. Spine (Phila Pa 1976). 2014; 39(19):1578-1583.

31. Yin $Q$, Xia $H$, Wu Z, et al. Surgical site infections following the transoral approach: a review of 172 consecutive cases. Clin Spine Surg. 2016;29(10):E502-E508.

32. Cantarella G, Mazzola RF, Benincasa A. A possible sequela of transoral approach to the upper cervical spine. Velopharyngeal incompetence. J Neurosurg Sci. 1998;42(1):51-55.

33. Di Lorenzo N. Transoral approach to extradural lesions of the lower clivus and upper cervical spine: an experience of 19 cases. Neurosurgery. 1989;24(1):37-42.

34. Hadley MN, Spetzler RF, Sonntag VK. The transoral approach to the superior cervical spine. A review of 53 cases of extradural cervicomedullary compression. J Neurosurg. 1989;71(1):16-23.

35. Menezes AH. Surgical approaches: postoperative care and complications "transoral-transpalatopharyngeal approach to the craniocervical junction." Childs Nerv Syst. 2008;24(10):1187-1193.

36. Bonney G, Williams JP. Trans-oral approach to the upper cervical spine. A report of 16 cases. J Bone Joint Surg Br. 1985;67(5): 691-698.

37. Jones DC, Hayter JP, Vaughan ED, Findlay GF. Oropharyngeal morbidity following transoral approaches to the upper cervical spine. Int J Oral Maxillofac Surg. 1998;27(4):295-298.

38. Hsu W, Wolinsky JP, Gokaslan ZL, Sciubba DM. Transoral approaches to the cervical spine. Neurosurgery. 2010;66(3 suppl): 119-125.

39. Merwin GE, Post JC, Sypert GW. Transoral approach to the upper cervical spine. Laryngoscope. 1991;101(7 pt 1):780-784.

40. Steinberger J, Skovrlj B, Lee NJ, et al. Surgical morbidity and mortality associated with transoral approach to the cervical spine. Spine (Phila Pa 1976). 2016;41(9):E535-E540.

41. Di Lorenzo N. Craniocervical junction malformation treated by transoral approach. A survey of 25 cases with emphasis on postoperative instability and outcome. Acta Neurochir (Wien). 1992; 118(3-4):112-116.

42. Castro-Marín F, Kendall JL. Diagnosis of Lemierre syndrome by bedside emergency department ultrasound. J Emerg Med. 2010;39 (4):436-439.

43. Nemakayala DR, Rai MP, Kavuturu S, Rayamajhi S. Atypical presentation of Lemierre's syndrome causing septic shock and acute respiratory distress syndrome. Case Rep Infect Dis. 2018; 2018:5469053.

44. Stam J. Thrombosis of the cerebral veins and sinuses. N Engl J Med. 2005;352(17):1791-1798. 
45. Schubert AD, Hotz MA, Caversaccio MD, Arnold A. Septic thrombosis of the internal jugular vein: Lemierre's syndrome revisited. Laryngoscope. 2015;125(4):863-868.

\section{Disclosures}

The authors report no conflict of interest concerning the materials or methods used in this study or the findings specified in this paper.

\section{Author Contributions}

Conception and design: Corvino. Acquisition of data: Corvino, Teodonno, Pagano. Analysis and interpretation of data: Corvino,
Teodonno, Pagano. Critically revising the article: Corvino, Mariniello, Maiuri. Reviewed submitted version of manuscript: Corvino, Maiuri. Approved the final version of the manuscript on behalf of all authors: Corvino. Administrative/technical/material support: Corvino, Teodonno, Pagano. Study supervision: Corvino, Mariniello, Maiuri.

\section{Correspondence}

Sergio Corvino: University "Federico II," Naples, Italy. sercorvino@ gmail.com. 